\title{
Mammalian Bite Injuries to the Hand and Their Management
}

\author{
Shilpa Jha ${ }^{1}$, Wasim S. Khan ${ }^{2}$ and Nashat A. Siddiqui ${ }^{*}, 1$ \\ ${ }^{I}$ Department of Trauma \& Orthopaedics, Kingston Hospital NHS Trust, Kingston, London, UK \\ ${ }^{2}$ University College London Institute of Orthopaedics and Musculoskeletal Sciences, Royal National Orthopaedic \\ Hospital, Stanmore, London, UK
}

\begin{abstract}
Bite wounds are a common form of hand injury with the potential to lead to severe local and systemic sequelae and permanent functional impairment. Mammalian bite wounds may be caused by a variety of animal class and species; injuries resulting from dogs, cats and humans are the most widely discussed and reported in the literature. Bite wounds may be contaminated with aggressive pathogens and the anatomical vulnerability of structures within the hand means that without early recognition and treatment with irrigation and antibiotics, alongside a low index of suspicion for deep structural involvement requiring formal surgical exploration and washout, the consequences of such injuries can be disastrous. We review the literature and discuss the epidemiology, pathophysiology and microbiology relating to these injuries, as well as clinical aspects including signs, symptoms, and management.
\end{abstract}

Keywords: Bite injuries, cat bite, dog bite, hand, human bite.

\section{INTRODUCTION}

Injuries to the hand can have a significant impact on the psychosocial well being of a patient [1]. Bite injuries to the hand are predominantly caused by mammals: dogs, cats and humans and represent a common emergency presentation. The estimated lifetime risk of being bitten by a domestic animal is around $50 \%$ [2], and these injuries account for $1-2 \%$ of emergency attendances in the US with an associated annual medical cost of over $\$ 100$ million [3]. Approximately $30-40 \%$ of all hand infections are attributable to human or animal bites [4]. Infectious sequelae of mammalian bite injuries occur most frequently in patients with injuries to the hand, and such injuries are more likely to require surgical treatment [5] than in other parts of the body. Human and animal bites account for $30-40 \%$ of traumatic hand infections [6]

Dog bite injuries are the most common, representing $80-90 \%$ of all mammalian bites [7], and most commonly affect the hand [5]. Around 250,000 people per year in the UK present with dog bite injuries to emergency and minor injuries units [8], including an estimated 5,000 postal workers per annum [9]. Dog bite injuries are more common in males and during the summer months [9]. In particular, elderly patients are more likely to suffer injuries to the hands, and more often require operative treatment and hospitalization [5]. Cat bite injuries are the second most common and show a higher incidence in females compared to dog bite injuries [11]. This is followed by human bites, which account for around 2-3\% of mammalian bites [12]. Human bites in adults are associated with alcohol

*Address correspondence to this author at the Department of Trauma \& Orthopaedics, Kingston Hospital NHS Trust, Kingston, London, UK; Tel: +44 (0) 208546 7711; Fax: +44 (0) 208546 7712;

E-mail: nashat75@gmail.com consumption, male gender, and have an increased incidence over the weekend and public holidays [13]. Dental personnel are also noted to have increased risk [14].

Although dogs, cats and humans are the most common sources of bite wounds, a wide range of mammals can cause bite injuries, with a wide geographical variation depending on local species populations, as well as risks associated with occupation and recreational hobbies. For example, monkey bite injuries are of increased prevalence amongst those who use them for medical research, as well as in regions that have large populations of free-roaming monkeys e.g. Gibraltar, Bali and India [15].

\section{PATHOPHYSIOLOGY}

Mammalian bite injuries to the hand are often of an occlusive nature. This occurs when the teeth close over the tissue, causing injuries ranging from superficial abrasions, puncture wounds, and lacerations to crushing injuries to tendons and muscle, tissue loss and avulsions, arterial and nerve injuries, fractures, dislocations and traumatic digit amputations $[2,13,16]$.

Canine jaws can have a bite force of up to $31,790 \mathrm{kPa}$ [8], with a significant potential for tissue damage. There may be a sharp injury to the skin and soft tissues from penetration of the teeth, as well as the potential for crush injury and fractures from the significant bite force. There is also the risk of a subtle injury to a tendon sheath, or penetration and inoculation into the deep palmar spaces, with a high risk of serious infection which may go unnoticed initially.

A notable subtype of human bite injury is the closed fist injury, often termed the "fight bite", occurring when the subject attempts to punch another person and their fist strikes their opponent's teeth. Studies have suggested that closed fist injuries account for around $50 \%$ of human bite injuries to 
the hand [17]. The metacarpophalangeal joint is at risk of injury. This commonly leads to penetration of the skin over the $2^{\text {nd }}, 3^{\text {rd }}, 4^{\text {th }}$ or $5^{\text {th }}$ metacarpal. Due to a relative paucity of tissue protecting the underlying tendon and joint capsule, this commonly results in a zone $\mathrm{V}$ extensor tendon injury. The force of impact may result in a fracture of the metacarpal neck, and occasionally an intra-articular fracture. Violation of the joint capsule leads to complications such as septic arthritis and osteomyelitis [18]. Furthermore, the mobility and retraction of the lacerated tendon allows for easy spread of the bacterial load away from the wound [19]. As the penetration of such tissues occurs when the fist is in a clenched position, it is vital to examine the wound in both open and clenched positions to evaluate the true depth of the wound [6]. This may require formal exploration under regional or general anaesthesia to track the path of the injury through different layers.

Infection is the most common complication of mammalian bite injuries to the hand, and may include cellulitis, tenosynovitis, septic arthritis and osteomyelitis. In a study examining bacterial isolates from 50 dog bite and 57 cat bite wounds, cultures were found to be predominantly polymicrobial, with a median of 5 bacterial isolates per culture, with $56 \%$ of wounds containing both aerobes and anaerobes. Pasteurella species were the most common isolates, followed by Streptococcus, Staphylococcus, Moraxella and Neisseria [20]. Studies suggest cat bites to have an increased risk of infection; a retrospective analysis of 525 bite wounds found $37.5 \%$ of cat bites became infected, compared to $14.9 \%$ of dog bites [21]. Cats, due to their sharper teeth, more often cause deep puncture wounds, as opposed to the higher likelihood of fracture or crush injury from dog bites. It has been postulated that the increased infection rate is due to the deep inoculation of bacteria through these narrow puncture wounds, which also have the tendency to rapidly seal off with haematoma, therefore inhibiting drainage and promoting infection and abscess formation [22]. Dog bite injuries can also be associated with Capnocytophaga canimorsus, an aggressive organism associate with disseminated sepsis and death, particularly in immunocompromised individuals [23].

Human bites are also at high risk of infection, with a reported infection rate of $48 \%$ in untreated hand bites [12]. The bacterial load of saliva has been estimated at $900 \times 10^{6}$ per $\mathrm{ml}$ [24] which may explain the considerable infection rate. A study examining cultures from 50 human bite injuries found that, like cat and dog bites, cultures are often polymicrobial (median number of isolates $=4$ ), with mixed anaerobes and aerobes in 54\% of wounds. Common organisms included Streptococcus anginosus (52\%), Fusobacterium nucleatum (32\%), Staphylococcus aureus (30\%), Eikenella corrodens (30\%) and Prevotella melaninogenica (22\%), and Candida species (8\%) [25].

In addition to pathogens from the biting animal, bacteria may also inoculate a wound by entering from the surrounding physical environment; thus bites occurring in marine environments are associated with pathogens such as Vibrio species, Plesiomonas shigelloides, Aeromonas hydrophila, and Pseudomonas species [26].

Injuries occurring in the farmyard environment are at very high risk of contamination with microbial organisms, many of which will be atypical bacteria, notably Actinomycetes, as well as highly virulent species which may result in significant necrosis and gas gangrene, such as Clostridia. If the patient has not had tetanus toxoid immunization in the last 5 years, tetanus prophylaxis should be administered, and those with large and grossly contaminated wounds should be considered for administration of tetanus immunoglobulin [27].

\section{INITIAL MANAGEMENT}

When taking a history from the patient it is pertinent to clearly document both the timing and mechanism of injury. It has been shown that a significant proportion of those with human bite injuries may give alternative presenting histories and only declare the true mechanism on direct questioning, due to a fear of prejudice if the patient confesses to violence being involved in sustaining the injury [28]. Possible causes given by patients presenting with such injuries include falls and work accidents, which would not immediately raise the suspicion of a laceration penetrating through different layers or being contaminated by atypical pathogens. Often no clear history can be obtained due to the patient being under the influence of alcohol or recreational drugs at the time of injury. Therefore, the level of suspicion for a significant injury must remain high until proven otherwise.

Patients with delayed presentation have been shown to have a significantly higher incidence of positive bacterial growth (100\% versus $54 \%$ in non-delayed patients), and a significantly higher likelihood of requiring surgical intervention ( $86 \%$ versus $48 \%$ in non-delayed patients) [29]. Closed-fist injuries in particular have been found at significantly increased likelihood of delayed presentation [19], increasing the risk of unfavourable outcome in this severe subtype of human bite injury. Clinical findings should be meticulously documented; dog bite injuries in particular are associated with litigation, both against animal owners (under the Dangerous Dogs Act 1991, United Kingdom), and clinicians for alleged mismanagement of the injury [9]. The history should also include risk factors for overwhelming infection and sepsis, such as being immunocompromised. Diabetic patients in particular are more likely to warrant admission and aggressive surgical treatment; studies have suggested that diabetic patients with hand infections have a $48 \%$ risk of requiring amputation compared with a $5 \%$ risk in non-diabetics [30].

Clinical examination should include thorough assessment of the depth of the wound, infectious signs and damage to associated structures such as tendons, nerves, blood vessels and bony injury $[31,32]$, and to exclude compartment syndrome [33]. Documentation may be augmented by the use of clinical photography. Dorsal wounds suggestive of clenched fist injury, associated with a history of altercation should be considered human bite injuries until proven otherwise. As previously mentioned, such wounds should be examined in both open and clenched fist position to give better demonstration of wound depth. Wounds overlying joints are of high risk of joint penetration; one study showed that $62 \%$ of all wounds overlying the metacarpo-phalangeal or proximal inter-phalangeal joints were found to have violated the underlying joint on surgical exploration [34]. A 
low threshold for formal surgical exploration is therefore indicated in such injuries.

Although evidence is unclear as to the risk of tetanus inoculation from mammalian bite wounds, randomised controlled trials to examine this would be considered unethical. It is therefore current practice that all patients who have no record of tetanus vaccination in the last 5 years should receive vaccine at presentation with mammalian bite injuries [35]. Patients bitten by dogs abroad should be considered for rabies vaccine and immunoglobulin, with further advice sought from relevant agencies such as the UK Health Protection Agency [9].

Human bite injuries should undergo assessment for risk of Hepatitis B and C transmission; inoculation has been described in case reports [36]. Administration of hepatitis B post-exposure prophylaxis is recommended by the UK Health Protection Agency for at risk groups, alongside testing for Hepatitis C, Hepatitis B and HIV at 6 weeks, 3 months and 6 months [37]. Risk of HIV transmission is thought to be low, however, it is recommended that advice from a specialist infectious disease team be sought regarding post-exposure prophylaxis, should the assailant have a known diagnosis of HIV or presence of an AIDS defining illness [37].

Prophylactic antibiotics are recommended for all bite injuries involving the hand - a Cochrane review of 8 randomised controlled trials found a statistically significant reduction in the rate of infection with a number needed to treat of just 4 [38]. Current NHS guidelines recommend empirical co-amoxiclav for 7 days as the $1^{\text {st }}$ choice antibiotic prophylaxis in dog, cat and human bite injuries [39]. Bites associated with marine environments require cover for associated organisms - ciprofloxacin has been suggested as a recommended agent for both fresh-water and salt-water wounds [40]. Patients who are systemically unwell or with deeper injuries are likely to warrant admission for intravenous antibiotics [41]. A suggested antibiotic regimen is 10-14 days in cellulitis, three weeks for tenosynovitis, four weeks for septic arthritis and six weeks for osteomyelitis, with conversion to oral antibiotics when the serum C-reactive protein is below $50 \mathrm{mg} / \mathrm{L} \mathrm{[9]}$.

\section{SURGICAL MANAGEMENT}

\section{Debridement and Lavage}

All mammalian bite wounds should undergo an initial thorough washout and decontamination, including copious irrigation with a minimum of 1 litre of saline [35]. A survey of 45 plastic surgery units in the UK found that this was performed in the emergency department in $91 \%$ of cases [42]. It has been suggested that local anaesthetic should be infiltrated into adjacent uninvolved skin to aid thorough initial washout and debridement [43]. However, infected tissue is associated with a lower $\mathrm{pH}$, an environment which leads to a reduced efficacy of local anaesthesic [44]. Therefore, the use of local anaesthetic infiltration may be of limited value if there is a clinical suspicion of infection.

Wounds often require further formal exploration, washout, and debridement in theatre. Factors affecting the need for surgery include depth, size, location, evidence of infection and damage to underlying structures,

A delay of over 24 hours before necessary surgical debridement has been shown to be a risk factor for the development of osteomyelitis [45]. Conversely, as expected, with early and thorough washout and debridement regimens, rates of infection can be reduced to $0.5 \%$ [9]. Formal surgical exploration and debridement should therefore occur in a timely fashion, ideally within 24 hours of initial injury.

Wounds should be extended proximally and distally as appropriate, to allow adequate examination of underlying structures and assessment of any damage to structures and evidence of infection. This is especially important with puncture wounds and clenched fist injuries where there may be deep inoculation of infection, and structural damage disproportionate to the overlying skin breakage. A study examining surgical findings in 191 patients taken to theatre with clenched fist injury found joint capsule violation in $67.8 \%$, tendon involvement in $20.3 \%$ and articular-bone indentation in $16.5 \%$ [46]. In a review of 12 cases of osteomyelitis of the hand after clenched fist injury, 11 patients were found to have a tooth mark in the bone or cartilage at the site of inoculation [45]. Thorough exploration including arthrotomy with thorough washout is therefore recommended for clenched fist injuries and other bites overlying joints to prevent septic arthritis and osteomyelitis.

\section{Fractures}

There is a paucity of evidence in the literature regarding timing of repair of associated structural injuries. Fractures, with or without associated dislocation, are the most common associated structural injury [47]. There is a lack of clear guidance specific to open fractures secondary to bite injuries, however, the general principles of open fracture management should be followed.

The Swanson, Szabo \& Anderson classification is specific to open fractures of the hand (distal to the carpus). Fractures secondary to bites would be classified as type II injuries, for which they advocate delayed closure. They found that the type of fixation does not affect infection rate, and the method of fixation should instead be influenced by the fracture pattern and rehabilitation needs [48]. However, a survey in the UK found that a large proportion of plastic surgery units do not provide definitive fixation of fractures during the initial washout (57\%), and this rises to $87 \%$ preferring delayed fixation if there is clinical suspicion of infection at initial washout [42]. Clearly there is still a fear of the metal implants becoming infected despite adequate debridement.

In other parts of the body, such as open tibia fractures, we know that adequate debridement and definitive fracture fixation, with soft tissue flaps if necessary, is the recommended treatment and is not associated with a high rate of infection $[49,50]$. Despite the hand being a highly vascular area, there is little soft tissue coverage of the bones, and therefore of any implants, and thus may still be prone to infection [51]. In addition, there are very real concerns about the amount of deep bacterial contamination. Despite evidence that primary fixation may safely be performed at the time of debridement, it is a decision that must be made 
by the clinician on a case-by-case basis, and there may be a role for external fixators $[52,53]$.

\section{Tendon, Nerve and Vascular Injuries}

Tendon and peripheral nerve injuries, particularly lacerations, are also found in such wounds. Peripheral nerve injuries are seen in around $2 \%$ of complicated bite cases [54]. Although there have not been any clear comparative studies in the literature, delayed reconstructions of tendons once clear of infection, staged if necessary, has been recommended [10, 40]. However, Evgeniou and colleagues found that $47 \%$ of plastic surgical units surveyed in the UK preferred to repair tendons and nerves at initial washout in theatre [42]. There is therefore a lack of clear consensus on whether tendon reconstruction should be primary or staged.

Tendon retraction may occur due to the delay between surgical debridement/lavage to treat the infection and definitive tendon reconstruction, but this may be minimised by preventing active or passive use of the hand, with formal immobilisation if necessary. Patients may require repeated washout and debridement in theatre alongside antibiotics depending on the clinical response.

Mammalian bites, in particular, may also be associated with vascular injuries; Akingba et al studied 371 patients presenting with dog bites and found $5.4 \%$ had a significant vascular injury requiring surgical intervention, with $85 \%$ of these injuries occurring in the upper limb. Injuries were repaired at initial debridement with primary anastomosis, bypass, ligation or a combination of techniques. Although there was a high rate of post-operative wound infection in this patient group (20\%), all limbs remained viable at 1-year follow-up [55].

\section{Post-Debridement Management}

Primary closure of bite injuries may be considered acceptable in superficial wounds in the head and neck where the relatively plentiful blood supply reduces the risk of infection, and there is a greater emphasis on optimal cosmesis. It is, however, relatively contra-indicated in injuries to the hand where studies have shown an infection rate of $25 \%$ after primary suture [21]. Bite injuries may be associated with skin and tissue loss requiring grafting and flap reconstruction. There is no clear guidance in the literature regarding staging of reconstruction in complex injuries where there may be multiple structures damaged, for example fractures with extensive skin and soft tissue loss.

Post-operatively, the injured hand should be elevated and immobilised in the position of function to reduce oedema, promote healing, and reduce pain, as in other hand infections. The period of immobilisation and commencement of rehabilitation exercises is dependent on factors associated with associated injured structures, such as fracture stability and union, and protection of tendon, nerve, or vascular repairs.

\section{CONCLUSION}

Bite injuries to the hand are a common presentation to emergency departments. Failure to recognise and adequately treat such injuries leads to a high risk of deep infection, structural damage and permanent loss of function, with the possibility of sepsis in high-risk patients. This is both due to the anatomical vulnerability of structures within the hand as well as polymicrobial inoculation with aggressive and atypical pathogens. Prophylactic antibiotics, a high index of suspicion of deep structural involvement and early and thorough washout and debridement are recommended to prevent the development of such serious limb-threatening sequelae.

There is a significant risk of persistent infection if bite injuries are primarily closed, and there must be a high index of suspicion for deep infection that would be better treated with debridement and washout, followed by either allowing the wound to heal or by delayed secondary closure. The environment of injury must be considered, e.g. farmyard environment, as these are associated with a high risk of bacterial contamination, particularly with atypical bacteria.

There have been few studies performed examining the optimal reconstruction methods and timing of reconstruction in associated injuries such as fracture, tissue loss, tendon, nerve and vascular lacerations. We recommend further studies to evaluate methods of surgical management of such injuries to provide optimal functional outcomes.

\section{CONFLICT OF INTEREST}

The authors confirm that this article content has no conflict of interest.

\section{ACKNOWLEDGEMENTS}

Declared none.

\section{REFERENCES}

[1] Malik A, Khan W. Recent advances and developments in hand surgery. Open Orthop J 2012; 6(1): 11-3.

[2] Nygaard M, Dahlin LB. Dog bite injuries to the hand. J Plast Surg Hand Surg 2011; 45(2): 96-101.

[3] Goldstein EJ. Bite wounds and infection. Clin Infect Dis 1992; 14(3): 633-8.

[4] Ouelett EA. Infections. In: Light TR, Ed. American Society of Surgery of the Hand: Hand Surgery Update 2. Rosemont, Ill.: Am Acad Orthop Surg 1999; pp. 411-2410.

[5] Pfortmueller CA, Efeoglou A, Furrer H, Exadaktylos AK. Dog bite injuries: primary and secondary emergency department presentations--a retrospective cohort study. Sci World J 2013; 2013: 393176.

[6] Ong YS, Levin LS. Hand Infections. Plast Reconstr Surg 2009; 124(4): $225 \mathrm{e}-33 \mathrm{e}$.

[7] Griego RD, Rosen T, Orengo IF, Wolf JE. Dog, cat, and human bites: a review. J Am Acad Dermatol 1995; 33(6): 1019-29.

[8] Thomas HF, Banks J. A survey of dog bites in Thanet. J R Soc Health 1990; 110: 173

[9] Morgan M, Palmer J. Dog Bites. BMJ 2007; 334(7590); 413-7.

[10] Lee YG, Jeong SH, Kim WK. An analytical study of Mammalian bite wounds requiring inpatient management. Arch Plast Surg 2013; 40(6): 705-10.

[11] Overall KL, Love M. Dog bites to humans--demography, epidemiology, injury, and risk. J Am Vet Med Assoc 2001; 218(12): 1923-34.

[12] Broder J, Jerrard D, Olshaker J, Witting M. Low risk of infection in selected human bites treated without antibiotics. Am J Emerg Med 2004; 22(1): 10-3. 
[13] Henry FP, Purcell EM, Eadie PA. The human bite injury: a clinical audit and discussion regarding the management of this alcohol fuelled phenomenon. Emerg Med J 2007; 24(7): 455-8.

[14] Carr MM. Human bites to the hand. J Can Dent Assoc 1995; 61(9): 782-4.

[15] Abrahamian FM, Goldstein EJ. Microbiology of animal wound infections. Clin Microbiol Rev 2011; 24(2): 231-46.

[16] Dire DJ. Emergence management of dog and cat bite wounds. Emerg Med Clin North Am 1992; 10(4): 719-36.

[17] Merchant RC, Zabbo CP, Mayer KH, Becker BM. Factors associated with delay to emergency department presentation, antibiotic usage and admission for human bite injuries. CJEM 2007; 9(6): 441-8.

[18] Faciszewski T, Coleman DA. Human bite wounds. Hand Clin 1989; 5(4): 561-9.

[19] Wienert P, Heiss J, Rinecker H, Sing A. A human bite. Lancet 1999; 354(9178): 572.

[20] Talan DA, Citron DM, Abrahamian FM, Moran GJ, Goldstein EJ. Bacteriologic analysis of infected dog and cat bites. Emergency Medicine Animal Bite Infection Study Group. N Engl J Med 1999; 340(2): 85-92.

[21] Aigner N, König S, Fritz A. Bite wounds and their characteristic position in trauma surgery management. Unfallchirurg 1996; 99(5): 346-50.

[22] Benson LS, Edwards SL, Schiff AP, Williams CS, Visotsky JL. Dog and cat bites to the hand: treatment and cost assessment. J Hand Surg Am 2006; 31(3): 468-73.

[23] Oehler RL, Velez AP, Mizrachi M, Lamarche J, Gompf S. Biterelated and septic syndromes caused by cats and dogs. Lancet 2009; 9(7): 439-47.

[24] Mantilla Gómez S, Danser MM, Sipos PM, Rowshani B, van der Velden U, van der Weijden GA. Tongue coating and salivary bacterial counts in healthy/gingivitis subjects and periodontitis patients. J Clin Periodontol 2001; 28(10): 970-8.

[25] Talan DA, Abrahamian FM, Moran GJ, Citron DM, Tan JO, Goldstein EJ. Emergency Medicine Human Bite Infection Study Group. Clinical presentation and bacteriologic analysis of infected human bites in patients presenting to emergency departments. Clin Infect Dis 2003; 37(11): 1481-9.

[26] Brook I. Management of human and animal bite wound infection: an overview. Curr Infect Dis Rep 2009; 11(5): 389-95.

[27] Angoules AG, Lindner T, Vrentzos G, Papakostidis C, Giannoudis PV. Prevalence and current concepts of management of farmyard injuries. Injury 2007; 38(Suppl 5): S27-34.

[28] Wallace CG, Robertson CE. Prospective audit of 106 consecutive human bite injuries: the importance of history taking. Emerg Med J 2005; 22(12): 883-4.

[29] Bach G, Shah NA, Mejia A, Weinzweig N, Brown A, Gonzalez MH. Upper extremity dog bite wounds and infections. J Surg Orthop Adv 2005; 14(4): 181-4.

[30] Gunther SF, Gunther SB. Diabetic hand infections. Hand Clin 1998; 14: 647-56.

[31] Griffin M, Hindocha S, Saleh M, Khan WS. An overview of the management of flexor tendon injuries. Open Orthop J 2012; 6(1): 28-35

[32] Haughton DN, Jordan D, Malahias M, Hindocha S, Khan WS. Principles of hand fracture management. Open Orthop J 2012; 6(1): 43-53.

[33] Malik AA, Khan WS, Chaudhry A, Ihsan M, Cullen NP. Acute compartment syndrome - a life and limb threatening surgical emergency. J Perioper Pract 2009; 19(5): 137-42.

[34] Phair IC, Quinton DN. Clenched fist human bite injuries. J Hand Surg Br 1989; 14(1): 86-7.
[35] Looke D, Dendle C. Bites (Mammalian). Clin Evid (Online) 2010; 2010: pii: 0914.

[36] Figueiredo JF, Borges AS, Martínez R, et al. Transmission of hepatitis $\mathrm{C}$ virus but not human immunodeficiency virus type 1 by a human bite. Clin Infect Dis 1994; 19(3): 546-7.

[37] Health Protection Agency North West. Guidance for the management of human bite injuries: Guidance for healthcare professionals on dealing with injuries where teeth break the skin. Health Protection Agency 2010. http://www.hpa.org.uk/webc/HPA webFile/HPAweb_C/1194947350692 [Accessed: January 23, 2014].

[38] Medeiros I, Saconato H. Antibiotic prophylaxis for mammalian bites. Cochrane Database Syst Rev 2001; (2): CD001738.

[39] National Institute for Health and Care Excellence. Bites - human and animal. National Institute for Health and Care Excellence Clinical Knowledge Summaries. http: //cks.nice.org.uk/biteshuman-and-animal [Accessed: January 23, 2014].

[40] Noonburg GE. Management of extremity trauma and related infections occurring in the aquatic environment. J Am Acad Orthop Surg 2005; 13(4): 243-53.

[41] Lane JCE, Mabvuure NT, Hindocha S, Khan WS. Current concepts of prophylactic antibiotics in trauma: a review. Open Orthop J 2012; 6: 511-7.

[42] Evgeniou E, Markeson D, Iyer S, Armstrong A. The management of animal bites in the United Kingdom. Eplasty 2013; 13: e27.

[43] Rosen RA. The use of antibiotics in the initial management of recent dog-bite wounds. Am J Emerg Med 1985; 3(1): 19-23.

[44] Becker DE, Reed KL. Essentials of local anesthetic pharmacology. Anesth Prog 2006; 53(3): 98-108.

[45] Gonzalez MH, Papierski P, Hall RF. Osteomyelitis of the hand after a human bite. J Hand Surg Am 1993; 18(3): 520-2.

[46] Patzakis MJ, Wilkins J, Bassett RL. Surgical findings in clenchedfist injuries. Clin Orthop Relat Res 1987; (220): 237-40.

[47] Benfield R, Plurad DS, Lam L, et al. The epidemiology of dog attacks in an urban environment and the risk of vascular injury. Am Surg 2010; 76(2): 203-5.

[48] Swanson TV, Szabo RM, Anderson DD. Open hand fractures: prognosis and classification. J Hand Surg Am 1991; 16(1): 101-7.

[49] BOAST 4: The Management of Severe Open Lower Limb Fractures; British Orthopaedic Association and British Association of Plastic, Reconstructive, and Aesthetic Surgeons Standard for Trauma - 2009; http://www.boa.ac.uk/LIB/LIBPUB/Documents/B OAST $\% 204 \% 20-\% 20$ The $\% 20$ Management $\% 20$ of $\% 20$ Severe $\% 200$ pen\%20Lower\%20Limb\%20Fractures.pdf [Accessed: February 21, 2014].

[50] Griffin M, Malahias M, Hindocha S, Khan WS. Update on the management of compound lower limb fractures. Open Orthop J 2012; 6: 518-24.

[51] Malik AA, Yousaf N, Khan WS, Ihsan M, Ravenscroft M. Fractures of the wrist and hand. J Perioper Pract 2010; 20(2): 48 54.

[52] Khan WS, Fahmy NRM. The S-Quattro in the management of intra-articular fractures of the fingers. J Hand Surg Br 2006; 31(1): 79-92.

[53] Bhamra JS, Khan WS, Pastides P. The use of the S-Quattro dynamic external fixator for the treatment of intra-articular phalangeal fractures: a review of the literature. Open Orthop J 2012; 6(1): 54-9.

[54] Hutson HR, Anglin D, Pineda GV, Flynn CJ, Russell MA, McKeith JJ. Law enforcement K-9 dog bites: injuries, complications, and trends. Ann Emerg Med 1997; 29(5): 637-42.

[55] Akingba AG, Robinson EA, Jester AL, et al. Management of vascular trauma from dog bites. J Vasc Surg 2013; 58(5): 1346-52. 